\title{
Fascia Thickness Increases Following Eccentric Exercise Leading to Delayed Onset Muscle Soreness
}

Sarah Tenberg ( $\nabla$ s.tenberg@hochschule-trier.de)

Fachhochschule Trier: Hochschule Trier https://orcid.org/0000-0001-7037-8111

Kazunori Nosaka

Edith Cowan University School of Medical and Health Sciences

Jan Wilke

Goethe-Universitat Frankfurt am Main Fachbereich 05 Psychologie und Sportwissenschaften

\section{Original Research Article}

Keywords: connective tissue, morphology, ultrasound, muscle damage, biceps brachii, pain

Posted Date: August 24th, 2021

DOl: https://doi.org/10.21203/rs.3.rs-826566/v1

License: (c) (1) This work is licensed under a Creative Commons Attribution 4.0 International License.

Read Full License 


\section{Abstract}

Background: The deep fascia fuses tightly with the skeletal muscle and, thus, may be damaged by eccentric loading.

Methods: To study its possible involvement in delayed onset muscle soreness, 11 healthy participants ( $\mathbb{\nabla}=$ $7 ; 24 \pm 2$ years) performed fatiguing dumbbell elbow flexor eccentric exercise (EE) for one arm and concentric exercise (CE) for the other arm in random order and with random arm allocation. Before, immediately after and 24-96 hours post-exercise, maximal voluntary isometric contraction torque of the elbow flexors (dynamometer), pressure pain (algometer), palpation pain (100-mm visual analog scale), biceps brachii fascia thickness and fascia/muscle mobility during passive movement (both highresolution ultrasound) were examined.

Results: Palpation pain, suggestive of DOMS, was greater after EE than CE, and maximal voluntary isometric contraction torque decreased greater after $E E$ than $C E(p<.05)$. Relative to $C E$, EE increased fascia thickness at $48(+17 \%), 72(+14 \%)$ and $96(+15 \%)$ hours post-exercise $(p<.05)$. At 96 hours post-EE, the increase in fascia thickness correlated with palpation pain $(r=.68 ; p<.05)$. Fascia mobility was not different between conditions, but compared to CE, muscle displacement increased at $24(+31 \%), 72$ $(+31 \%)$, and $96(+41 \%)$ hours post-EE $(p<.05)$.

Conclusion: Collectively, these results suggest an involvement of the deep fascia in delayed onset muscle soreness.

\section{Key Points}

- Fascia thickness increases after eccentric exercise of the elbow flexors

- Increase of fascia thickness partly correlated with palpation pain

- Deep fascia may contribute to the pathogenesis of DOMS; however, evidence of a cause-effect relationship is currently lacking

\section{Background}

The collagenous connective tissue exhibits an intimate mechanical relationship with the skeletal muscle. This is most obvious for the tendon which links muscular and bony structures. However, reducing force transmission to these two tissues is a fallacy [1-4]. Biomechanical experiments have shown that the forces measured at the distal and proximal tendon during muscle lengthening are not equal [1]. It is assumed that the observed proximo-distal force difference stems from mechanical interactions with nontendinous connective tissues [1]. In some muscles, up to $80 \%$ of the fibers do not span the entire distance between origin and insertion [5]. Instead, fusion of adjacent fibers, and with this, an indirect connection between both ends of the muscle is created by the endomysium [5]. It has been demonstrated that mechanical interactions between neighboring muscle fibers occur due to translaminar shearing [6]. 
Hence, intramuscular force transmission is realized through the fibers themselves and their endomysium. A similar architecture-function relationship can be observed for the perimysium and the deep fascia. Ultrastructural analyses have shown that the perimysium transmits lateral forces between muscle fiber bundles due to direct tissue continuity [7]. Likewise, the deep fascia interacts with the underlying muscle via fibrous tissue expansions and direct fiber insertions $[8,9]$.

A recent systematic review with meta-analysis has shown that clinically diagnosed muscle injuries rarely affect the muscle tissue in an isolated manner [10]. In contrast, structural lesions mostly occur in the connective tissue, namely the myotendinous junction and the deep fascia. Considering that eccentric overload represents the main mechanism of injury, it has been hypothesized that the connective tissue, via its numerous links to muscular structures, absorbs and dampens excessive elongating forces [10]. Similar to muscle injury, delayed onset muscle soreness (DOMS) is predominantly induced by unaccustomed exercises with eccentric contractions. It could therefore be speculated that the associated tissue overstretch produces micro-lesions in the fascia which contribute to the development of DOMS at 24-72 hours post-exercise. This assumption is supported by both histological and experimental studies [11-18]. Due to its dense innervation and equipment with sensory receptors and free nerve endings that act as algogenic nociceptors $[11,19]$, fascia appears to be of fundamental relevance in pain perception [13-18]. Schilder [17] investigated the pain sensitivity of different structures injecting hypertonic saline into the lumbar fascia and the erector spinae muscle, and showed that the irritation of the connective tissue evoked stronger pain responses than the muscle. Gibson [14] performed a similar experiment inducing DOMS by eccentric exercise in one leg. While injection of hypertonic saline into the exercised muscle did not increase pain sensitivity compared to the inactive control leg, a strong increase (exercised vs. control leg) was registered upon irritation of the fascia. Collectively, these findings suggest that the fascia rather than muscle fiber damage seems to cause DOMS. This is supported by data showing that structural muscle damage does not or only weakly correlate with the magnitude of DOMS [20-22].

Although fascia has a high nociceptive potential and may be vulnerable to mechanical overload (i.e., eccentric contractions), evidence on connective tissue involvement in DOMS is still scarce. The aim of the present study, therefore, was to investigate morphological changes in the biceps brachii fascia after eccentric exercise of the elbow flexors. Since it is known that concentric contractions do not induce DOMS, morphological and functional changes in the fascia were compared between eccentric and concentric contractions. We hypothesized that, e.g. due to edema and swelling, fascia thickness would increase and fascia mobility would decrease after eccentric exercise only, and that the magnitude of the changes in the fascia would be associated with the magnitude of DOMS.

\section{Methods}

\subsection{Study design and ethics}

A randomized, controlled crossover trial with two exercise conditions was performed. At least 72 hours after a familiarization session, each participant visited the laboratory on 5 consecutive days. On day 1 , 
eccentric and concentric exercise (randomized order and arm allocation) of the elbow flexors was performed using a dumbbell. Outcome measures (see below) were assessed immediately before (base), immediately after (post 0 ) and every 24 hours up to 96- hours post-exercise (post 24, 48, 72, and 96). The study was approved by the Edith Cowan University Human Research Ethics Committee, and adhered to the Guidelines of Good Clinical Practice as well as the Declaration of Helsinki. All participants provided a written informed consent prior to the study participation.

\subsection{Participants}

Eleven (seven male and four female) healthy active students $(24.3 \pm 1.9 \mathrm{y} ; 167 \pm 6 \mathrm{~cm} ; 67.2 \pm 8.1 \mathrm{~kg} ; 293$ \pm 145 min sporting activity per week) volunteered to participate in the present study. The Edinburgh Handedness Inventory - Short Form [23] showed that all participants were right-handed. None of the participants had performed regular upper arm resistance training in the preceding six months. All individuals were healthy, not reporting any orthopedic, cardiovascular, neurological, endocrine or psychiatric diseases. Intake of anti-inflammatory or analgesic medicine was prohibited during or on the days before the experiment.

\subsection{Determination of one-repetition maximum (1-RM)}

Immediately before the exercise session (day 2), the one repetition maximum (1-RM) was measured using dumbbell curls (one arm for eccentric exercise, and the other arm for concentric exercise) according to the randomization (e.g., left arm eccentric, right arm concentric). This was done because a first bout of eccentric exercise could protect against muscle damage in subsequent bouts of exercise [16, 24]. After a warm-up with a light ( $50 \%$ of the estimated 1-RM) dumbbell (10 elbow flexion and extension movements), the participants performed either one isolated eccentric (lowering the dumbbell) or concentric (lifting the dumbbell) contraction with a load of approximately $80 \%$ of the estimated 1-RM. For the 1-RM assessment, each participant sat on a preacher arm-curl bench to stabilize their shoulder. For the eccentric 1-RM, the participant was instructed to lower the dumbbell from $90^{\circ}$ elbow flexion to full extension in three seconds, and for the concentric 1-RM, the participant lifted the dumbbell from full extension to $90^{\circ}$ flexion in three seconds. After each attempt, a one-minute rest was applied, and the procedure was repeated increasing the weight based on the perceived effort of the participant until a failed attempt occurred. All participants required less than five attempts for each arm to determine the 1$\mathrm{RM}$.

\subsection{Exercise protocols}

The participants performed 6 sets of 10 repetitions of eccentric contractions for one arm and concentric contractions of the elbow flexors for the other arm. Repetition duration was 3 seconds and the inter-set interval was 2 minutes. The exercises were performed with a dumbbell, using $80 \%$ of the individual 1-RM [25]. After each repetition, the investigator assisted in returning the dumbbell to its starting position to ensure eccentric-only or concentric-only contractions were performed with the load [26]. If necessary, the investigator carefully supported the participant to complete the protocol. After each set, the participants 
were asked to rate the effort of the exercise using the rating of perceived exertion (CR-10) scale ranging from 1 anchoring "extremely easy," to 10, "extremely hard" [27].

\subsection{Outcome measures}

The following measurements were taken from the exercised arm before (base), immediately after (excluding muscle pain measures) and 24, 48, 72 and 96 hours after exercise. To avoid possible influences of circadian rhythm, examinations were performed at approximately the same time of the day for each participant.

\subsubsection{Maximal voluntary isometric contraction (MVIC) torque}

MVIC torque was measured using an isokinetic dynamometer (Biodex system 3, Biodex medical, Shirley, NY, USA). Data were recorded and stored via the LabChart Pro software (v8.1.5, ADInstruments) to a laptop computer. Each participant was positioned in supine on a therapy bed having the elbow joint aligned with the dynamometer's axis of rotation (Fig. 1). The elbow joint of the examined arm was positioned at $90^{\circ}$ flexion, and both shoulders were stabilized on the therapy bed. Following a brief warmup, each participant was instructed to flex the elbow joint as fast and strong as possible against the stationary lever arm for 3 seconds. Verbal encouragement was provided to elicit maximal effort for each attempt. Two attempts with a one-minute rest were performed. The average peak force, which could be maintained for 1 second, was considered as the MVIC torque and the mean of both measurements was used for further analysis [28, 29].

\subsubsection{Pressure pain threshold (PPT)}

PPT of the biceps brachii of the exercised arm was measured using an electronic algometer (Somedic AB, Sollentuna, Sweden). Each participant lay on a therapy bed in a relaxed supine position with the forearm being slightly elevated by a soft pad. The probe of the algometer (diameter of $1 \mathrm{~cm}$ ) was placed on the biceps brachii muscle belly ( $9 \mathrm{~cm}$ above the elbow crease), and compressive force was gradually increased at a rate of approximately $50 \mathrm{kPa} / \mathrm{s}$ until the participant reported the first sensation of pain. The value $(\mathrm{kPa})$ corresponding to the force applied at this moment was recorded. After a 10-second rest interval, the procedure was repeated twice, and the average of the two measures was used for further analysis.

\subsubsection{Palpation pain}

Muscle pain upon palpation of the elbow flexors was assessed by a 100-mm visual analogue scale (VAS), anchoring 0 being no pain and 100 being worst possible pain [30,31]. Each participant was in the same position as that of PPT, and the measurement was taken from the same place as that of PPT $(9 \mathrm{~cm}$ above the elbow crease). The investigator palpated the muscle of each participant with two fingers in longitudinal orientation [32]. The participants were asked to indicate the level of perceived pain by marking it on the VAS. Pressure was always applied by the same investigator and kept as constant as 
possible (approximately $400 \mathrm{kPa}$ ) between days and participants [28]. The measurement site was marked on the skin with a semi-permanent marker and renewed on a daily basis.

\subsubsection{Fascia thickness}

A high-resolution ultrasound (US) device (Aloka ProSound F75, Hitachi Healthcare, Tokyo, Japan) with an $8.0 \times 1.5 \mathrm{~cm}$ linear array transducer was used (frequency range of $7.5 \mathrm{MHz}$, display depth of $3.0 \mathrm{~cm}$, dynamic range of $60 \mathrm{~dB}$ and image gain of 50) to measure fascia thickness. Each participant was positioned in supine with the examined arm being attached to the lever arm of the isokinetic dynamometer at $35^{\circ}$ flexion (Fig. 1). The ultrasound probe was positioned longitudinally on the biceps muscle belly (with the center of the probe $9 \mathrm{~cm}$ above the elbow crease). The investigator ensured to stabilize the probe on the skin with minimal pressure in order to avoid compressing the tissue. Three static images were taken when the deep fascia was clearly recognizable as a hyperechoic region overlying the muscle. To ensure identical positioning during follow-up measurements, the US-transducer location was marked on the skin with a semi- permanent marker and renewed every day.

Fascia thickness was calculated using ImageJ (Image J $1.52 \mathrm{k}$ software, USA). Within each US image, five regions of interest (ROIs) at equidistant points were selected for the thickness measurement (Fig. 2a). The average of the five ROls in each of the three images was chosen to determine the fascia thickness.

\subsubsection{Fascia and muscle displacement}

High-resolution ultrasound imaging was also used to evaluate fascia and muscle displacement during passive movements of the elbow joint of the exercised arm. Each participant was positioned as explained for the MVIC torque measure. The linear array ultrasound transducer was positioned longitudinally on the belly of the biceps brachii with the center of the probe at $9 \mathrm{~cm}$ above the elbow crease, and fixed with elastic bandages and tape, ensuring the muscle was not compressed. The isokinetic dynamometer passively moved the elbow joint three times between $90^{\circ}$ flexion and full extension at an angular velocity of $5 \%$ s (33). Previous studies have shown that reflexive muscle contraction does not occur at this velocity $[34,35]$. Participants were instructed to remain completely relaxed, avoiding any voluntary muscle activity. In order to familiarize the participants with the device and measurement conditions, a warm-up of three flexion-extension cycles was performed prior to the actual measurements [36, 37]. Video recordings, depicting fascia and muscle tissue, were made via ultrasound at $10 \mathrm{~Hz}$ during the passive movement. Additionally, elbow joint position [ $\left.{ }^{\circ}\right]$, relative to the neutral zero position, was recorded from the dynamometer signal to the laptop computer. Ultrasound videos were cut into extension and flexion parts of the three repetitions using the synchronized joint position. If a participant could not reach full extension in the follow-up days due to increased muscle stiffness, all videos of this participant were cut to the lowest achieved extension angle.

The maximal horizontal displacement of biceps brachii fascia and muscle was quantified using a frameby-frame cross correlation algorithm proposed by Dilley [38]. To determine tissue displacement, rectangular ROls were selected in the ultrasound videos. The used software [38] computes the correlation 
coefficient between the pixel gray levels of the consecutive frames. The pixel shift with the highest correlation coefficient represents the tissue displacement between the two successive frames. Three equidistant ROls were defined in the fascia starting $2.5 \mathrm{~cm}$ from the myotendinous junction. Additionally, three equidistant ROls were defined in the muscle on the same level. Horizontal tissue displacement was calculated by calculating the mean of three ROls and three repetitions for the corresponding tissue (Fig. $2 \mathrm{~b}$ and $2 \mathrm{c})$.

\subsection{Statistical analyses}

All data were tested for normal distribution using a Shapiro Wilk test and inference statistics were applied as appropriate. Baseline values were compared between conditions (eccentric vs. concentric) using a paired t-test or a Wilcoxon test. To examine the possible influence of exercise order (eccentric or concentric arm first), baseline values of participants starting with the respective conditions were compared by means of independent $t$ tests or Mann-Whitney-U tests. As an additional check, we used point biserial correlation or contingency tables with Cramer- $V$ to detect potential association of pre-post differences and treatment order.

Prior to the main analysis comparing between eccentric and concentric exercise for changes in the dependent variables over time, sphericity was checked by a Mauchly's test and the normal distribution of the residuals was examined by a Shapiro-Wilk test. If requirements were met, we performed 2 -factorial ANOVAs (condition * time). Resulting effect sizes $\left(\eta^{2}\right)$ were interpreted as small (0.01), medium (0.06) or large (0.14) according to Cohen (1988) [39]. In case of main effects for time, Bonferroni-Holm adjusted post hoc tests (paired t-test) were performed. Effect size interpretation followed the recommendations of Cohen (1988), distinguishing small ( $d=0.2$ to 0.5 ), medium ( 0.5 to 0.8 ) or large ( 0.8 or higher) effects [39]. In case of violations of the assumptions for parametric testing, Kubinger's bifactorial rank variance analyses with Bonferroni-Holm corrected post-hoc Wilcoxon tests were computed. We also investigated possible correlations between fascia thickness, fascia displacement, muscle displacement and palpation pain by a Pearson or Spearman correlation analysis. According to Cohen (1980), correlation coefficients (CC) were interpreted a small ( $r=0.1$ to 0.3$)$, medium ( 0.3 to 0.5$)$ or large ( 0.5 and higher) [40].

All analyzes were performed with SPSS 26 (SPSS Inc., Chicago, Illinois, USA) and BiAs Statistics (version 11.10, Goethe University, Frankfurt am Main, Germany). The level of significance was set to $a=0.05$.

\section{Results}

All eleven participants completed the exercise protocol. However, the muscle/fascia displacement data of one participant had to be excluded due to an erroneous synchronization of the isokinetic testing system and the ultrasound device. No differences in the baseline values between the two exercise sessions were evident, and no intervention order effects were found $(p>.05)$.

\subsection{MVIC torque}


The $2 \times 5$ bifactorial-rank-variance analysis for MVIC showed no interaction effect $(p>0.05)$, but significant main effects for condition $(p<0.001)$ and time $(p<0.001)$. The post-hoc analysis for condition showed significantly lower values for EE than CE at post $0(r=-0.80 ; p=0.008)$, post24 $(r=-0.80 ; p=0.008)$ and post48 $(r=-0.86 ; p=0.004)$ even after Bonferroni-Holm correction (post0 $=0.024$; post24 $=0.032$; post $48=0.02 ;$ Fig. 3$)$. Likewise, also the post-hoc analysis for time identified differences at all measurements $(p<0.05)$, even after Bonferroni-Holm correction.

\section{2. $P P T$}

The 2x4 ANOVA for PPT did not reveal interactions or main effects for condition ( $p>0.05)$, but a main effect for time $\left(\eta^{2}=0.239 ; p=0.04\right)$. Post-hoc testing showed significant differences between post-24 and post-72 ( $d z=-0.47 ; p=0.04)$, post -24 and post-96 $(d z=-0.62 ; p=0.009)$ as well as post 48 and post96 $(d z=-0.49 ; p=0.03)$. However, after Bonferroni-Holm correction, these differences were insignificant (Fig. 4).

\subsection{Palpation pain}

The $2 \times 4$ bifactorial-rank-variance analysis for pain upon palpation revealed no interaction effect $(p>$ 0.05). However, a significant main effect was found for time $(p=0.01)$ and condition $(p<0.001$, Fig. 10). Post-hoc tests showed a significant difference between EE and CE for all time points [post24 $(r=-0.89 ; p=$ 0.003), post48 $(r=-0.75 ; p=0,013)$, post72 $(r=-0.70 ; p=0.021)$ and post96 $(r=-0.69 ; p=0.022)]$, even after the Bonferroni Holm correction [post24 $(p=0.012)$, post48 $(p=0.039)$, post72 $(p=0.042)$ and post96 $(p=$ 0.022)]. Collectively, these data suggest that EE consistently induced stronger pain (Fig. 4).

\subsection{Fascia thickness}

The 2x5 ANOVA for fascia thickness revealed an interaction effect of time and condition $\left(\eta^{2}=0.323 ; p=\right.$ $0.003)$. The post-hoc tests showed differences between $E E$ and $C E$ at post24 $(d z=0.74 ; p=0.034)$, post48 $(d z=1.32 ; p=0.001)$, post72 $(d z=0.86 ; p=0.016)$ and post96 $(d z=0.96 ; p=0.010)$. After BonferroniHolm correction, only the differences at post48 $(d z=1.32 ; p=0.005)$, post72 $(d z=0.86 ; p=0.048)$ and post96 $(\mathrm{dz}=0.96 ; \mathrm{p}=0.040)$ were significant (Fig. 5 and Table 1).

\subsection{Fascia and muscle displacement}

The $2 \times 5$ bifactorial-rank-variance analysis revealed no interaction effect for either fascia or muscle displacement ( $p>0.05$; Fig. 6 , Table 1). However, regarding muscle displacement, a significant main effect for condition was found $(p=0.003)$. Post-hoc tests showed a significant difference between $E E$ and $C E$ at post24 $(T=-3.582 ; p=0.006)$, post48 $(T=-3.398 ; p=0.009)$, post72 $(T=-2.984 ; p=0.018)$ and post96 $(T=-4.495 ; p=0,001)$. After Bonferroni-Holm correction, most comparisons remained significant (post24: $p=0.024$, post48: $p=0.027$, post72: $p=0.036$, post $96: p=0.005$ ) although one (post $0: p=0.051$ ) failed to meet the significance threshold.

\subsection{Correlations}


Spearman's rank correlation showed no significant association of pre-post differences in VAS and fascia thickness for CE. Contrarily, increased fascia thickness following EE correlated strongly with palpation pain at 96-hours post-exercise $(r=0.682, p=0.021)$. Additionally, at 72-hours post- exercise, there was a tendency for a correlation $(p=0.051)$ with a high positive correlation coefficient $(r=0.60)$.

Regarding tissue displacement measures, only muscle displacement correlated with VAS at post24 $(r=-0.675 ; p=0.032)$ and post96 $(r=-0.685 ; p=.029)$ after CE. Yet, there was a tendency for an association of muscle displacement and VAS pain at post48 after $E E(r=0.626 ; p=0.053)$.

\section{Discussion}

Skeletal muscle fiber damage and inflammation have often been discussed as primary sources of DOMS [41-44]. However, the pain occurring after eccentric exercise is not or only weakly related to the structural muscle damage identified in related experiments [20-22]. The present study demonstrated that eccentric exercise increased fascia thickness to a considerable degree and this increase correlated with participant's pain sensations. Taken together, our findings suggest a relevant contribution of the deep fascia to the pathogenesis of DOMS, although a cause-effect relationship is yet to be established.

Changes in pain sensation after eccentric exercise were already evaluated and shown to be related to the fascia in two previous studies $[14,16]$. Injection of hypertonic sodium solution and electrical stimulation of the connective tissue caused higher intensities and durations of pain than the irritation of the underlying muscle itself $[14,16]$. However, the mechanisms underlying these observations have not been scrutinized. Solomonow [45] proposed a model describing the degradation of viscoelastic tissue after prolonged high-load exercise. Briefly, it suggests that repeated mechanical strain leads to micro-damage to collagen fibers, which, in turn, can trigger inflammation. Indirect signs of collagen breakdown after eccentric exercise have been observed in the form of increased total serum collagen $[46,47]$ and type IV collagen concentration [48]. Although a cause-effect relationship cannot be derived from our data, it is assumed that the increases in fascia thickness reflect tissue inflammation and/or edema triggered by collagenous micro-failure of the muscular connective tissue, which would be in line with data describing the vulnerability of fascia in muscle injury [10].

We observed an increase in passive muscle tissue displacement after eccentric exercise, but no such change was found in the fascia. Previous studies have revealed that the sliding of the deep fascia against the muscle is a significant functional soft tissue property, which, if altered, may induce muscle pain [13]. We expected that damage of the fascia following eccentric exercise might negatively affect both total fascial mobility and tissue gliding. While the former could not be affirmed by our data, muscle displacement increased after eccentric exercise but not after concentric exercise. This was in line with the findings from Lau et al. [49], who found a significant increase (50\%) in biceps brachii myotendinous junction displacement during eccentric elbow flexor contractions from the first to the 10 th set in the first bout. After a second bout of the same exercise performed four weeks later, no changes in the displacement were evident. Since DOMS typically occurs after the first bout of eccentric exercise but not 
after the second bout, the authors concluded that the change in muscle length affected the magnitude of muscle damage. With regard to our data, taking together both, the increased muscle displacement in the passive movement after eccentric exercise and the constant fascia mobility suggest that the deep fascia was unable to adapt to the higher muscle movement. Yet, as this observation was not correlated with the magnitude of DOMS, future research is warranted to conclusively judge the relevance of an altered muscle-fascia mobility ratio.

Our findings may have direct implications for exercise professionals designing programs for recreational and elite athletes. Particularly in competitive sports, where rapid recovery and restoration of performance are key factors for success. In view of the increasing evidence suggesting an involvement of the deep fascia in DOMS, treatments providing tissue-specific stimuli could accelerate recovery. Recently, Clifford et al. [50] found a moderate-magnitude reduction of DOMS after supplementation with collagen peptides which would fit with an assumed existence of micro-damage. A plethora of studies, moreover, demonstrated that massage [51-53] and foam rolling [54-57] alleviated DOMS. Finally, it has been repeatedly shown that the deep fascia generally adapts to mechanical loading $[36,58]$. Future research elucidating exercise paradigms addressing the deep fascia could therefore open new frontiers in the prevention and therapy of DOMS.

Some methodological issues need to be discussed. During measurements, no electromyographic recordings were made in order to verify that the movement induced by the isokinetic dynamometer was purely passive. However, this can be assumed with a relatively high degree of certainty as previous studies showed that such continuous passive motion does not provoke muscle activity [34,38]. Another issue relates to the sample size. Our study was explorative in nature and the identification of betweencondition effects with only 11 (10 for tissue displacement) participants suggests a relevant implication of fascia morphology in DOMS. However, with regard to insignificant parameters (i.e. fascia mobility), future studies with larger sample sizes may still observe small-magnitude effects. Finally, it is worth mentioning that a wash-out phase between conditions (concentric and eccentric exercise) was omitted. A carryover effect could thus have influenced the measurements from a theoretical point of view; however, it is unlikely that the results were largely affected by a carryover effect (e.g., contralateral repeated bout effect) to make the comparison between eccentric and concentric exercise conditions unreasonable.

\section{Conclusions}

Eccentric exercise increased fascia thickness, which was associated with the magnitude of DOMS. These findings support and expand the available evidence suggesting an involvement of the extramuscular connective tissue in the pathogenesis of DOMS. Future studies should (1) clarify the potential association of eccentric loading, connective tissue damage, local inflammation and DOMS, and (2) develop and test specific exercise paradigms addressing the deep fascia for recovery.

\section{Abbreviations}


1-RM: One repetition maximum

CC: Correlation coefficient

CE: Concentric exercise

DOMS: Delayed onset muscle soreness

EE: Eccentric exercise

MVIC: Maximal voluntary isometric contraction

PPT: Pressure pain threshold

ROIs: Regions of interest

US: Ultrasound

VAS: Visual analogue scale

\section{Declarations}

Ethics approval and consent to participate: The study was conducted according to the guidelines of the Declaration of Helsinki and approved by the Edith Cowan University Human Research Ethics Subcommittee Medical \& Health Sciences, Joondalup, Australia (No. 2019-00727-NOSAKA). Informed consent was obtained from all subjects involved in the study.

Consent for publication Not applicable

Availability of data and material: The datasets used and analysed during the current study are available from the corresponding author on reasonable request

Competing Interests: Sarah Tenberg, Kazunori Nosaka and Jan Wilke declare that they have no competing interests.

Funding: This research received no external funding.

Authors' Contributions: Conceptualization, J.W.; methodology, J.W.; K.N. and S.T.; software, J.W. and S.T.; validation, J.W.; K.N. and S.T; formal analysis, J.W.; S.T.; investigation, S.T.; resources, K.N.; data curation, S.T.; writing-original draft preparation, S.T and J.W.; writing-review and editing, J.W.; K.N. and S.T.; visualization, S.T.; supervision, J.W. and K.N.; project administration, K.N. All authors have read and agreed to the published version of the manuscript.

Acknowledgments: The authors thank Roshni Fernando, Suresh Rathnayake, Isuru Rathnayaka, Ayantha Adikari, Manu Perera, Sumudu Muthunayaka, Pasindu Silva, Heshandri Dharmadasa, Ushani 
Dissanayake and Janathri Hiranya for their assistance in data collection.

\section{References}

1. Wilke J, Schleip R, Yucesoy CA, Banzer W. Not merely a protective packing organ? A review of fascia and its force transmission capacity. J Appl Physiol. 2018 Jan;124(1):234-44.

2. Yucesoy CA, Baan GC, Koopman BHFJM, Grootenboer HJ, Huijing PA. Pre-strained epimuscular connections cause muscular myofascial force transmission to affect properties of synergistic EHL and EDL muscles of the rat. J Biomech Eng. 2005 Oct;127(5):819-28.

3. Krause F, Wilke J, Vogt L, Banzer W. Intermuscular force transmission along myofascial chains: a systematic review. J Anat. 2016 Jun;228(6):910-8.

4. Huijing PA, Baan GC. Myofascial force transmission via extramuscular pathways occurs between antagonistic muscles. Cells Tissues Organs. 2008;188(4):400-14.

5. Hijikata T, Ishikawa H. Functional morphology of serially linked skeletal muscle fibers. Acta Anat (Basel). 1997;159(2-3):99-107.

6. Purslow PP, Trotter JA. The morphology and mechanical properties of endomysium in series-fibred muscles: variations with muscle length. J Muscle Res Cell Motil. 1994 Jun;15(3):299-308.

7. Passerieux E, Rossignol R, Letellier T, Delage JP. Physical continuity of the perimysium from myofibers to tendons: involvement in lateral force transmission in skeletal muscle. J Struct Biol. 2007 Jul;159(1):19-28.

8. Stecco C, Macchi V, Porzionato A, Duparc F, De Caro R. The fascia: the forgotten structure. Ital J Anat Embryol = Arch Ital di Anat ed Embriol. 2011;116(3):127-38.

9. Stecco C, Gagey O, Macchi V, Porzionato A, De Caro R, Aldegheri R, et al. Tendinous muscular insertions onto the deep fascia of the upper limb. First part: anatomical study. Morphologie. 2007 Mar;91(292):29-37.

10. Wilke J, Hespanhol L, Behrens M. Is It All About the Fascia? A Systematic Review and Meta-analysis of the Prevalence of Extramuscular Connective Tissue Lesions in Muscle Strain Injury. Orthop J Sport Med. 2019 Dec;7(12):2325967119888500.

11. Stecco C, Gagey O, Belloni A, Pozzuoli A, Porzionato A, Macchi V, et al. Anatomy of the deep fascia of the upper limb. Second part: study of innervation. Morphologie. 2007;91(292):38-43:

10.1016/j.morpho.2007.05.002.

12. Mense S. Innervation of the thoracolumbar fascia. Eur J Transl Myol. 2020;29(3):151-8: 10.4081/ejtm.2019.8297.

13. Langevin HM, Fox JR, Koptiuch C, Badger GJ, Greenan- Naumann AC, Bouffard NA, et al. Reduced thoracolumbar fascia shear strain in human chronic low back pain. BMC Musculoskelet Disord. 2011 Dec 19;12(1):203: 10.1186/1471-2474-12-203.

14. Gibson W, Arendt-Nielsen L, Mizumura TTK, Graven-Nielsen T. Increased pain from muscle fascia following eccentric exercise: Animal and human findings. Exp Brain Res. 2009 Apr;194(2):299-308: 
10.1186/1471-2474-12-203.

15. Klingler, Werner; Jäger, Heike; Pedro, Maria T.; Schleip R. Faszien als Ursache von Schmerzsyndromen. In: Herbert, Michael; Meißner W, editor. Aktuelle Schmerzmedizin. Ecomed Medizin; 2014.

16. Lau WY, Blazevich AJ, Newton MJ, Wu SSX, Nosaka K. Changes in electrical pain threshold of fascia and muscle after initial and secondary bouts of elbow flexor eccentric exercise. Eur J Appl Physiol. 2015 May;115(5):959-68.

17. Schilder A, Hoheisel U, Magerl W, Benrath J, Klein T, Treede R-D. Sensory findings after stimulation of the thoracolumbar fascia with hypertonic saline suggest its contribution to low back pain. Pain. 2014 Feb;155(2):222-31.

18. Stecco C, Stern R, Porzionato A, Macchi V, Masiero S, Stecco A, et al. Hyaluronan within fascia in the etiology of myofascial pain. Surg Radiol Anat. 2011 Dec;33(10):891-6.

19. Mense S. Innervation of the thoracolumbar fascia. Eur J Transl Myol. 2019 Aug;29(3):8297.

20. Nosaka K, Newton M, Sacco P. Delayed-onset muscle soreness does not reflect the magnitude of eccentric exercise-induced muscle damage. Scand J Med Sci Sport. 2002 Dec 1;12(6):337-46: 10.1034/j.1600-0838.2002.10178.x.

21. Nurenberg P, Giddings CJ, Stray-Gundersen J, Fleckenstein JL, Gonyea WJ, Peshock RM. MR imaging - Guided muscle biopsy for correlation of increased signal intensity with ultrastructural change and delayed-onset muscle soreness after exercise. Radiology. 1992 Sep 1;184(3):865-9:

10.1148/radiology.184.3.1509081.

22. Hotfiel T, Freiwald J, Hoppe M W, Lutter C, Forst R, Grim C, Bloch w, Hüttel M, Hiess R. Advances in Delayed-Onset Muscle Soreness (DOMS): Part I: Pathogenesis and Diagnostics. Sportverletz Sportschaden. 2018;32(4):243-50: 10.1055/A-0753-1884.

23. Veale JF. Edinburgh Handedness Inventory - Short Form: A revised version based on confirmatory factor analysis. Laterality. 2014;19(2):164-77: 10.1080/1357650X.2013.783045.

24. Chen TC, Yang TJ, Huang MJ, Wang HS, Tseng KW, Chen HL, et al. Damage and the repeated bout effect of arm, leg, and trunk muscles induced by eccentric resistance exercises. Scand J Med Sci Sport. 2019 May 1;29(5):725-35: 10.1111/sms.13388.

25. Nguyen D, Brown LE, Coburn JW, Judelson DA, Eurich AD, Khamoui A V, et al. Effect of delayed-onset muscle soreness on elbow flexion strength and rate of velocity development. J strength Cond Res. 2009 Jul;23(4):1282-6.

26. Chen TC, Nosaka K. Responses of elbow flexors to two strenuous eccentric exercise bouts separated by three days. J strength Cond Res. 2006 Feb;20(1):108-16.

27. Morishita S, Yamauchi S, Fujisawa C, Domen K. Rating of Perceived Exertion for Quantification of the Intensity of Resistance Exercise. Int J Phys Med Rehabil. 2013;1(9):172.

28. Lau WY, Muthalib M, Nosaka K. Visual analog scale and pressure pain threshold for delayed onset muscle soreness assessment. J Musculoskelet Pain. 2013 Dec;21(4):320-6. 
29. Nosaka K, Newton M. Concentric or eccentric training effect on eccentric exercise-induced muscle damage. Med Sci Sports Exerc. 2002 Jan;34(1):63-9.

30. Haefeli M, Elfering A. Pain assessment. Eur Spine J. 2006;15: 10.1007/s00586-005-1044-x.

31. Turk DC, Melzack R. The measurement of pain and the assessment of people experiencing pain. In: Handbook of pain assessment, 3rd ed. New York, NY, US: The Guilford Press; 2011. p. 3-16.

32. Lau WY, Blazevich AJ, Newton MJ, Wu SSX, Nosaka K. Assessment of Muscle Pain Induced by Elbow-Flexor Eccentric Exercise. J Athl Train. 2015 Nov;50(11):1140-8.

33. Cruz-Montecinos C, González Blanche A, López Sánchez D, Cerda M, Sanzana-Cuche R, CuestaVargas A. In vivo relationship between pelvis motion and deep fascia displacement of the medial gastrocnemius: Anatomical and functional implications. J Anat. 2015 Nov 1;227(5):665-72: 10.1111/joa.12370.

34. Gajdosik RL. Influence of age on calf muscle length and passive stiffness variables at different stretch velocities. Isokinet Exerc Sci. 1997 Jan 1;6(3):163-74.

35. Lamontagne A, Malouin F, Richards CL. Viscoelastic behavior of plantar flexor muscle-tendon unit at rest. J Orthop Sports Phys Ther. 1997;26(5):244-52: 10.2519/jospt.1997.26.5.244.

36. Krause F, Wilke J, Niederer D, Vogt L, Banzer W. Acute effects of foam rolling on passive stiffness, stretch sensation and fascial sliding: A randomized controlled trial. Hum Mov Sci. 2019 Oct 1;67: 10.1016/j.humov.2019.102514.

37. Wilke J, Debelle H, Tenberg S, Dilley A, Maganaris C. Ankle Motion Is Associated With Soft Tissue Displacement in the Dorsal Thigh: An in vivo Investigation Suggesting Myofascial Force Transmission Across the Knee Joint. Front Physiol. 2020;11:180.

38. Dilley A, Greening J, Lynn B, Leary R, Morris V. The use of cross-correlation analysis between highfrequency ultrasound images to measure longitudinal median nerve movement. Ultrasound Med Biol. 2001;27(9):1211-8: 10.1016/S0301-5629(01)00413-6.

39. Cohen J. Statistical Power Analysis for the Behavioral Sciences. Hoboken: Taylor and Francis; 1988.

40. Cohen, L.; Manion L. Research Methods in Education. London: Croom Helm; 1980.

41. Friden J, Sjostrom M, Ekblom B. Myofibrillar damage following intense eccentric exercise in man. Int J Sports Med. 1983;4(3):170-6: 10.1055/s-2008-1026030.

42. Friden J, Kjorell U, Thornell LE. Delayed muscle soreness and cytoskeletal alterations: An immunocytological study in man. Int J Sports Med. 1984;5(1):15-8: 10.1055/s-2008-1025873.

43. Fridén J, Lieber RL. Eccentric exercise-induced injuries to contractile and cytoskeletal muscle fibre components. In: Acta Physiologica Scandinavica. Acta Physiol Scand; 2001. p. 321-6: 10.1046/j.1365-201X.2001.00834.x.

44. Newham DJ, McPhail G, Mills KR, Edwards RHT. Ultrastructural changes after concentric and eccentric contractions of human muscle. J Neurol Sci. 1983;61(1):109-22: 10.1016/0022510X(83)90058-8. 
45. Solomonow M. Neuromuscular manifestations of viscoelastic tissue degradation following high and low risk repetitive lumbar flexion. J Electromyogr Kinesiol; 2012; 22: 155-75:

10.1016/j.jelekin.2011.11.008.

46. Brown S, Day S, Donnelly A. Indirect evidence of human skeletal muscle damage and collagen breakdown after eccentric muscle actions. J Sports Sci. 1999 May;17(5):397-402: 10.1080/026404199365911.

47. Takagi R, Ogasawara R, Tsutaki A, Nakazato K, Ishii N. Regional adaptation of collagen in skeletal muscle to repeated bouts of strenuous eccentric exercise. Pflugers Arch Eur J Physiol. 2016 Sep 1;468(9):1565-72: 10.1007/s00424-016-1860-3.

48. Koskinen SO, Ahtikoski AM, Komulainen J, Hesselink MK, Drost MR, Takala TE. Short-term effects of forced eccentric contractions on collagen synthesis and degradation in rat skeletal muscle. Pflugers Arch Eur J Physiol. 2002;444(1-2):59-72: 10.1007/s00424-002-0792-2.

49. Lau WY, Blazevich AJ, Newton MJ, Wu SSX, Nosaka K. Reduced muscle lengthening during eccentric contractions as a mechanism underpinning the repeated-bout effect. Am J Physiol Integr Comp Physiol. 2015 May 15;308(10):R879-86: 10.1007/s00424-002-0792-2.

50. Clifford T, Ventress M, Allerton DM, Stansfield S, Tang JCY, Fraser WD, et al. The effects of collagen peptides on muscle damage, inflammation and bone turnover following exercise: a randomized, controlled trial. Amino Acids. 2019 Apr 1;51(4):691-704: 10.1007/s00726-019-02706-5.

51. Frey Law LA, Evans S, Knudtson J, Nus S, Scholl K, Sluka KA. Massage Reduces Pain Perception and Hyperalgesia in Experimental Muscle Pain: A Randomized, Controlled Trial. J Pain. 2008 Aug;9(8):714-21: 10.1016/j.jpain.2008.03.009.

52. Micklewright $D$. The effect of soft tissue release on delayed onset muscle soreness: A pilot study. Phys Ther Sport. 2009 Feb;10(1):19-24: 10.1016/j.ptsp.2008.09.003.

53. Zainuddin Z, Newton M, Sacco P, Nosaka K. Effects of massage on delayed-onset muscle soreness, swelling, and recovery of muscle function. J Athl Train. $2005 \mathrm{Jul} ; 40(3): 174-80$.

54. Jay K, Sundstrup E, Søndergaard SD, Behm D, Brandt M, Særvoll CA, et al. Specific and cross over effects of massage for muscle soreness: randomized controlled trial. Int J Sports Phys Ther. 2014 Feb;9(1):82-91.

55. Macdonald GZ, Button DC, Drinkwater EJ, Behm DG. Foam rolling as a recovery tool after an intense bout of physical activity. Med Sci Sports Exerc. 2014 Jan;46(1):131-42:

10.1249/MSS.0b013e3182a123db.

56. Pearcey GEP, Bradbury-Squires DJ, Kawamoto JE, Drinkwater EJ, Behm DG, Button DC. Foam rolling for delayed-onset muscle soreness and recovery of dynamic performance measures. J Athl Train. 2015 Jan 1;50(1):5-13: 10.4085/1062-6050-50.1.01.

57. Romero-Moraleda B, Touche R La, Lerma-Lara S, Ferrer-Peña R, Paredes V, Peinado AB, et al. Neurodynamic mobilization and foam rolling improved delayed-onset muscle soreness in a healthy adult population: A randomized controlled clinical trial. PeerJ. 2017;2017(10): 10.7717/peerj.3908. 
58. Zügel M, Maganaris CN, Wilke J, Jurkat-Rott K, Klingler W, Wearing SC, et al. Fascial tissue research in sports medicine: from molecules to tissue adaptation, injury and diagnostics: consensus statement Consensus statement. Br J Sport Med. 2018;52:1497: 10.1136/bjsports-2018-099308.

\section{Table}

Table 1

Values for fascia thickness, fascia displacement and muscle displacement in the corresponding condition at the different times including all participants.

\begin{tabular}{|c|c|c|c|c|c|c|}
\hline & Baseline & post & $24 \mathrm{~h}$ & $48 \mathrm{~h}$ & $72 \mathrm{~h}$ & $96 \mathrm{~h}$ \\
\hline \multicolumn{7}{|c|}{ Fascia thickness [mm] \# } \\
\hline \multirow[t]{2}{*}{ EE } & $\begin{array}{l}0.6629 \\
\pm\end{array}$ & $\begin{array}{l}0.7028 \pm \\
0.0820\end{array}$ & $\begin{array}{l}0.7272 \pm \\
0.0729\end{array}$ & $\begin{array}{l}0.7415 \pm \\
0.0826\end{array}$ & $\begin{array}{l}0.7307 \pm \\
0.0609\end{array}$ & $\begin{array}{l}0.7296 \pm \\
0.0768\end{array}$ \\
\hline & 0.0996 & $(+7 \%)$ & $(+11 \%)$ & $(+13 \%)$ & $(+12 \%)$ & $(+11 \%)$ \\
\hline \multirow[t]{2}{*}{ CE } & $\begin{array}{l}0.7003 \\
\pm\end{array}$ & $\begin{array}{l}0.7075 \pm \\
0.0667\end{array}$ & $\begin{array}{l}0.6921 \pm \\
0.0646\end{array}$ & $\begin{array}{l}0.6703 \pm \\
0.068\end{array}$ & $\begin{array}{l}0.6877 \pm \\
0.0796\end{array}$ & $\begin{array}{l}0.6742 \pm \\
0.0735\end{array}$ \\
\hline & 0.0596 & $(+1 \%)$ & $(-1 \%)$ & $(-4 \%)$ & $(-2 \%)$ & $(-4 \%)$ \\
\hline $\begin{array}{l}\text { \% difference } \\
\text { EE-CE }\end{array}$ & & $+6 \%$ & $+12 \%$ & $+17 \%$ & $+14 \%$ & $+15 \%$ \\
\hline \multicolumn{7}{|c|}{ Fascia displacement [mm] § } \\
\hline \multirow[t]{2}{*}{ EE } & 4.54 & 5.09 & 3.95 & 3.41 & 2.89 & 2.81 \\
\hline & $\begin{array}{l}(2.61- \\
8.57)\end{array}$ & $\begin{array}{l}(0.13- \\
17.90)\end{array}$ & $\begin{array}{l}(-1.10- \\
16.60)\end{array}$ & $\begin{array}{l}(2.19- \\
18.52)\end{array}$ & $\begin{array}{l}(1.29- \\
15.85)\end{array}$ & $(1.16-20.5)$ \\
\hline \multirow[t]{2}{*}{ CE } & 6.29 & 4.95 & 6.42 & 6.38 & 5.03 & 5.40 \\
\hline & $\begin{array}{l}(4.46- \\
10.47)\end{array}$ & $\begin{array}{l}(1.26- \\
10.61)\end{array}$ & $\begin{array}{l}(-0.37- \\
10.36)\end{array}$ & $\begin{array}{l}(0.61- \\
11.08)\end{array}$ & $\begin{array}{l}(0.96- \\
10.73)\end{array}$ & $\begin{array}{l}(0.25- \\
10.66)\end{array}$ \\
\hline \multicolumn{7}{|c|}{ Muscle displacement [mm] \# } \\
\hline \multirow[t]{3}{*}{$\mathrm{EE}$} & $41.28 \pm$ & $49.50 \pm$ & $52.87 \pm$ & $52.73 \pm$ & $51.72 \pm$ & $53.62 \pm$ \\
\hline & 7.72 & 15.72 & 9.88 & 12.33 & 9.49 & 12.55 \\
\hline & & $(+21 \%)$ & $(+30 \%)$ & $(+38 \%)$ & $(+31 \%)$ & $(+40 \%)$ \\
\hline \multirow[t]{3}{*}{ CE } & $54.98 \pm$ & $48.96 \pm$ & $53.83 \pm$ & $48.57 \pm$ & $51.41 \pm$ & $54.23 \pm$ \\
\hline & 11.25 & 9.51 & 11.45 & 9.50 & 9.35 & 10.69 \\
\hline & & $(-9 \%)$ & $(-1 \%)$ & $(-6 \%)$ & $(+/-0 \%)$ & $(-1 \%)$ \\
\hline $\begin{array}{l}\text { \% difference } \\
\text { EE-CE }\end{array}$ & & $+30 \%$ & $+31 \%$ & $+44 \%$ & $+31 \%$ & $+41 \%$ \\
\hline
\end{tabular}


Values are given in $\mathrm{mm}$. The percentage indicate the increase/decrease compared to baseline. $(\mathrm{EE}=$ eccentric exercise; $\mathrm{CE}=$ concentric exercise, $\#=$ mean \pm standard deviation, $\S=$ median (minimum maximum)).

\section{Figures}

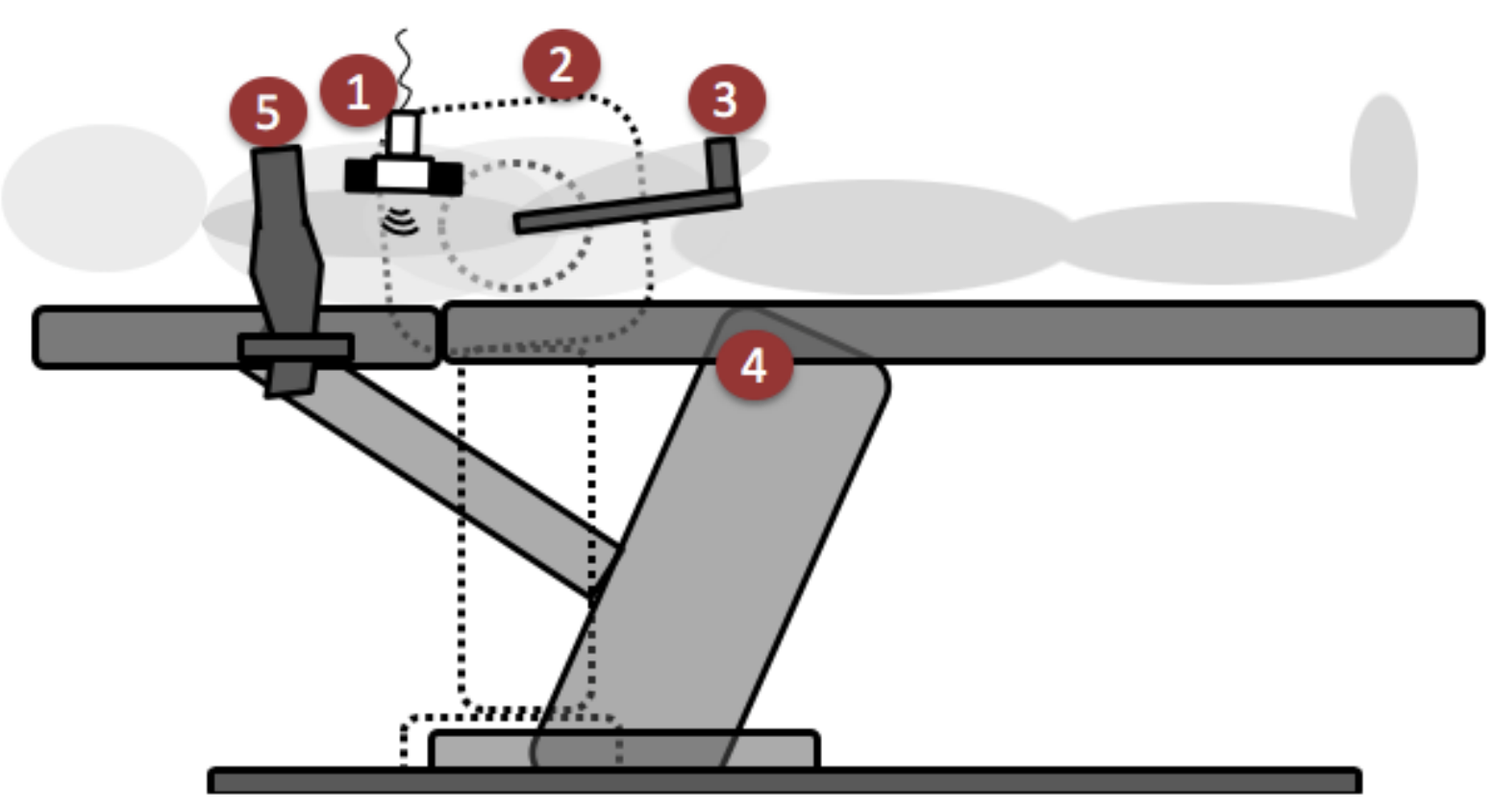

\section{Figure 1}

Position of a participant and placement of the isokinetic dynamometer (shown in dashed lines, since it was located behind the therapy table) for the measurement of fascia thickness. (1) ultrasound transducer, (2) isokinetic dynamometer in $17.5^{\circ}$ rotation and $35^{\circ}$ elbow flexion, (3) arm bracket of the isokinetic dynamometer, (4) therapy table (5) shoulder stabilization. 
a)

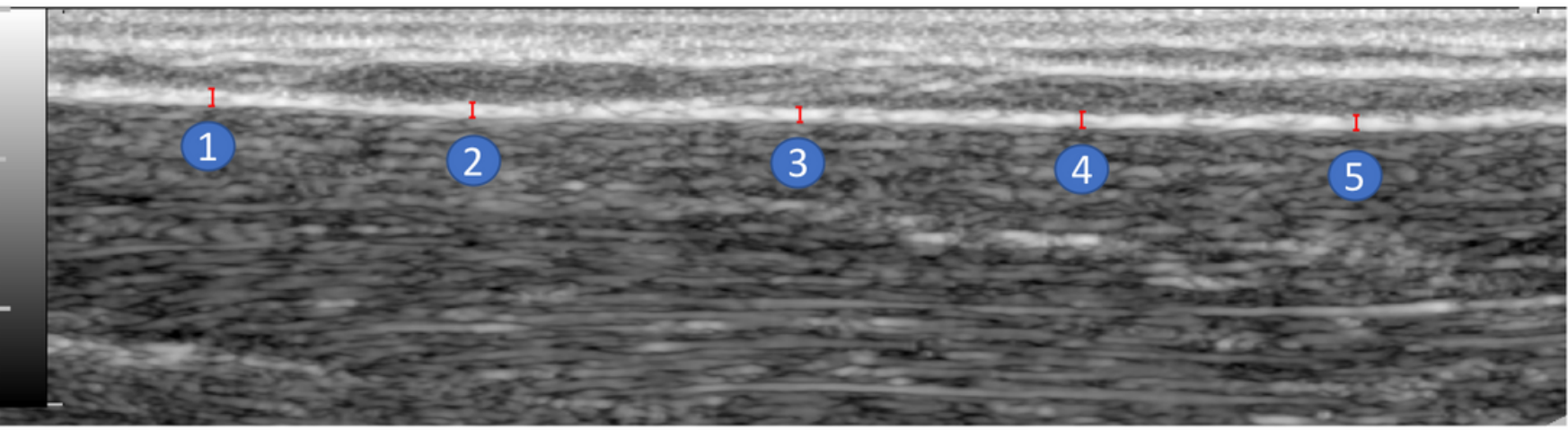

b)

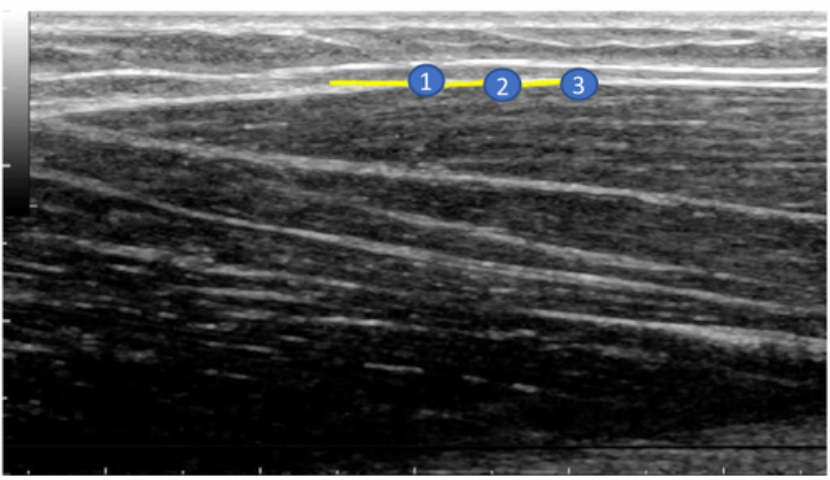

c)

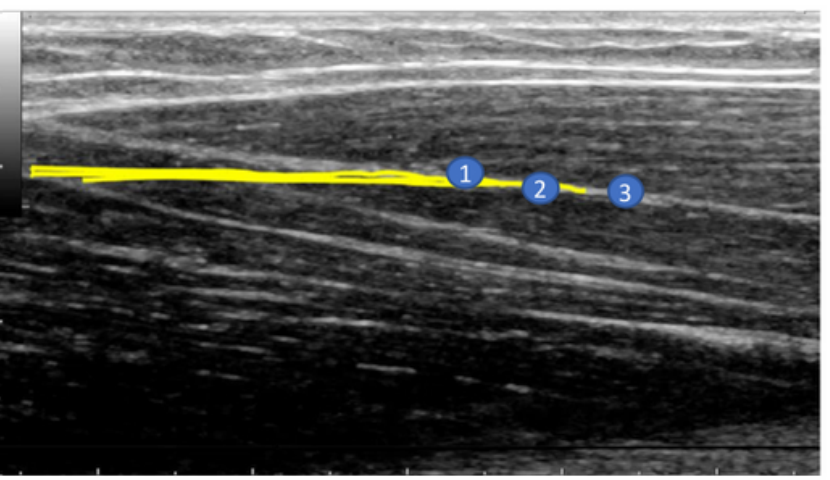

Figure 2

Fascia thickness was measured at 5 equidistant locations (a). Tissue mobility was measured in the fascia (b) and the muscle (c) using three regions of interest. 


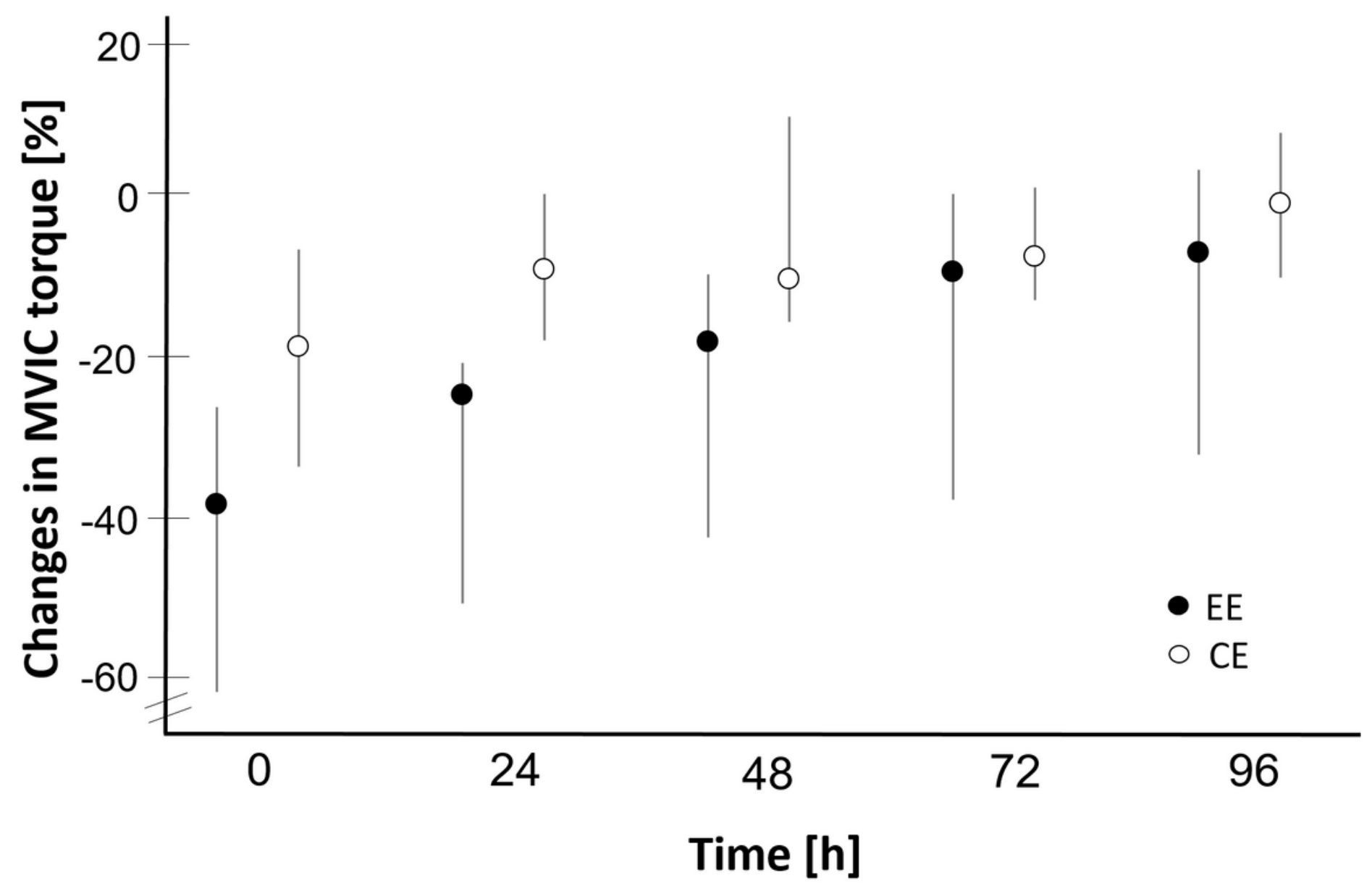

Figure 3

Changes in maximal voluntary isometric contraction (MVIC) torque after exercise. Figure shows nonparametric $95 \%$ confidence intervals. $\mathrm{EE}=$ eccentric exercise, $\mathrm{CE}=$ concentric exercise.

- $\mathrm{EE}$

O CE
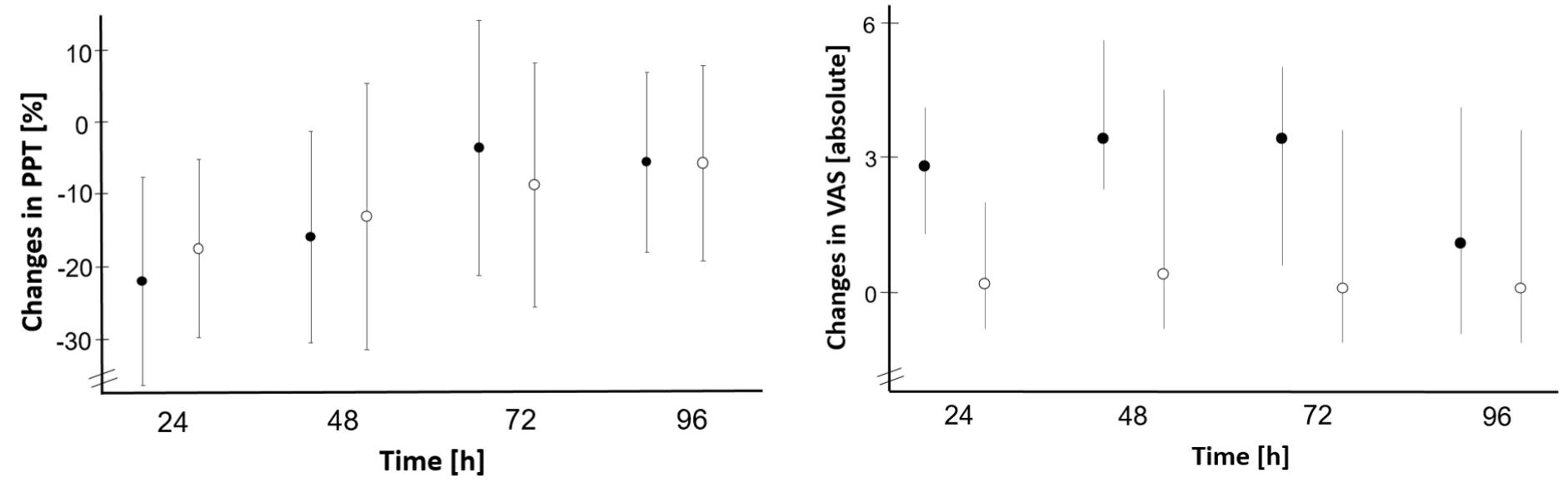

Figure 4 
Changes in pressure pain threshold (PPT) and pain on palpation (visual analogue scale, VAS) pain after exercise. Figure shows parametric (PPT) and non-parametric (VAS) 95\% confidence intervals. EE = eccentric exercise, $\mathrm{CE}=$ concentric exercise.

- $\mathrm{EE}$

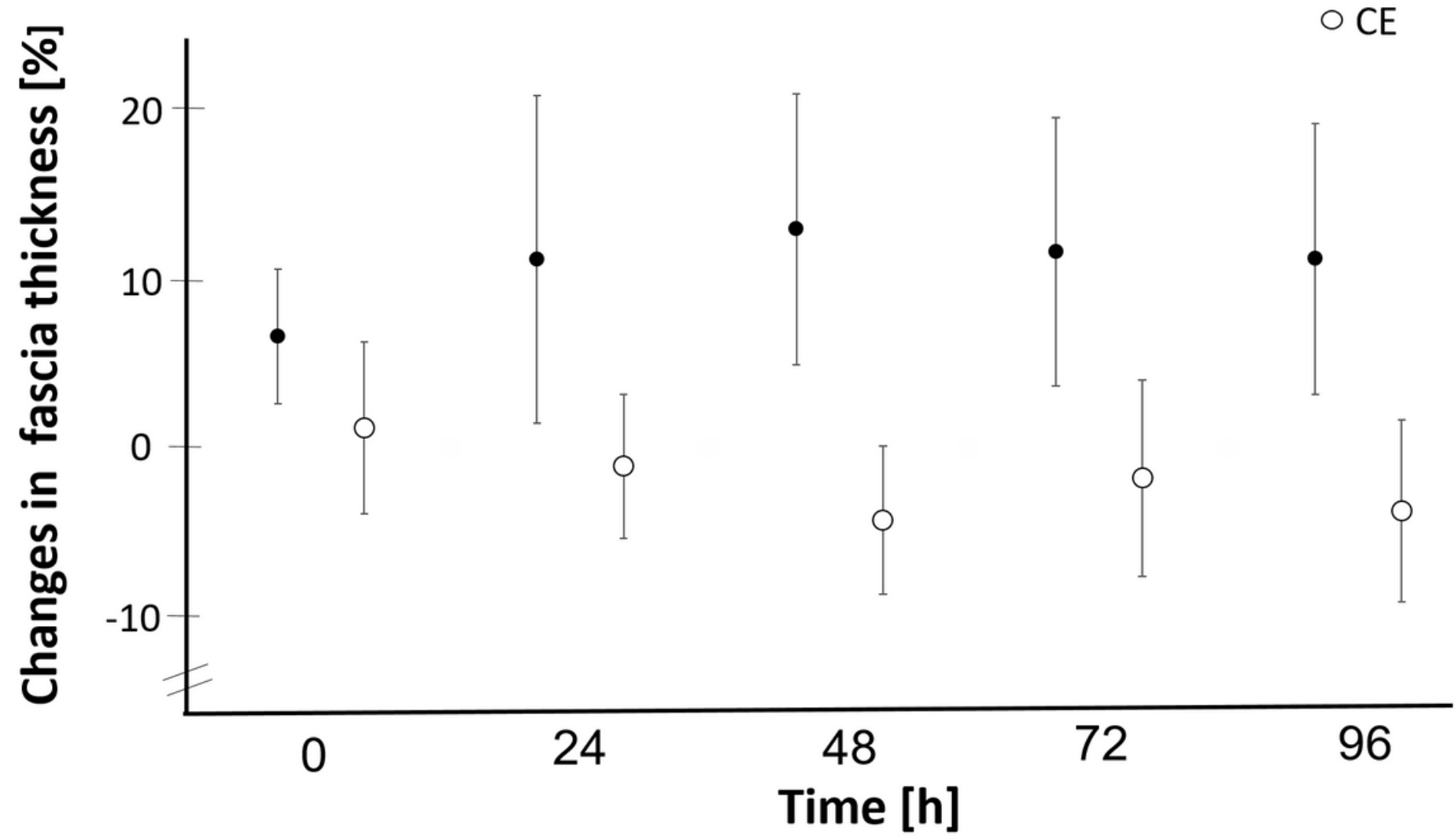

Figure 5

Changes in fascia thickness after exercise. Figure shows parametric $95 \%$ confidence intervals. $E E=$ eccentric exercise $\mathrm{CE}=$ concentric exercise.

- $\mathrm{EE}$

O CE
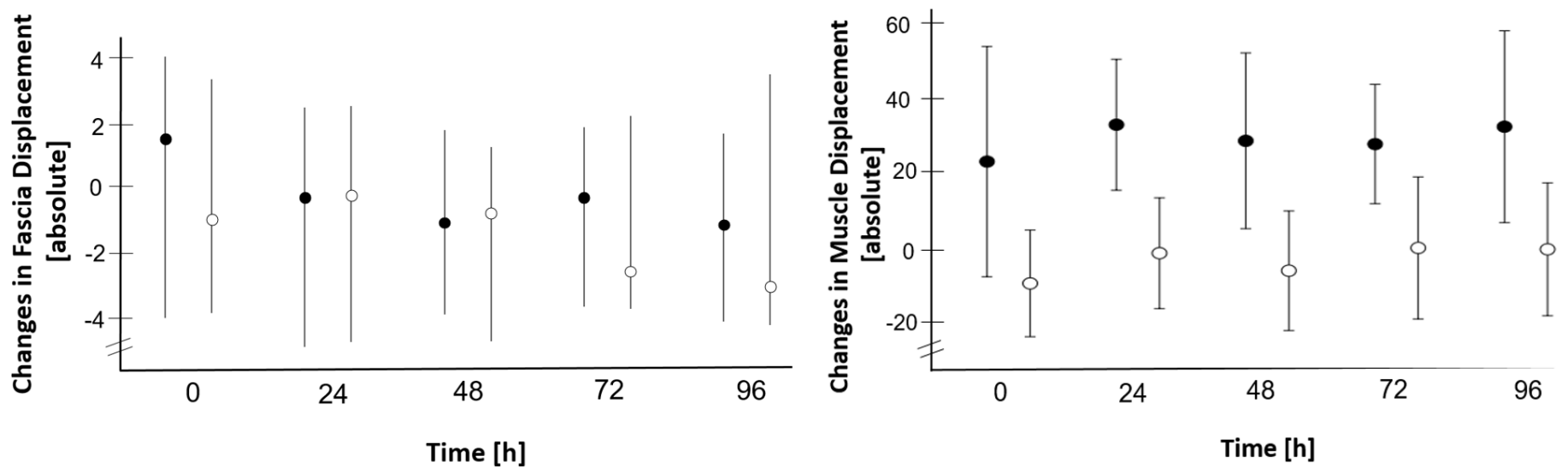

Figure 6 
Changes in fascia and muscle displacement after exercise. Figure shows non-parametric (fascia displacement) and parametric (muscle displacement) 95\% confidence intervals. $E E=$ eccentric exercise, $\mathrm{CE}=$ concentric exercise 